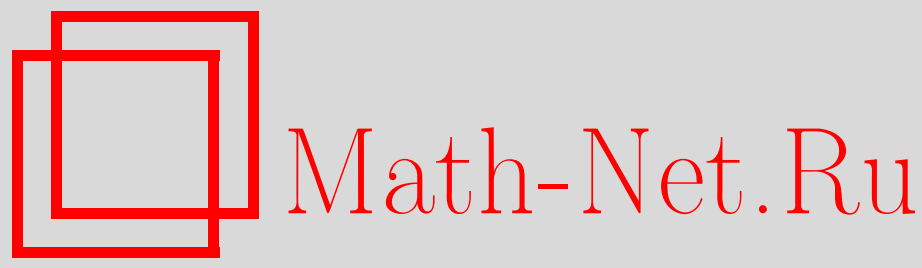

Ю. В. Матиясевич, Гипотеза Римана в терминах собственных чисел специальных ганкелевых матриц, Совр. пробл. матем., 2016, выпуск 23, 87-101

DOI: https://doi.org/10.4213/spm64

Использование Общероссийского математического портала Math-Net.Ru подразумевает, что вы прочитали и согласны с пользовательским соглашением http://www . mathnet.ru/rus/agreement

Параметры загрузки:

IP : 54.197 .217 .227

26 апреля 2023 г., 18:00:57

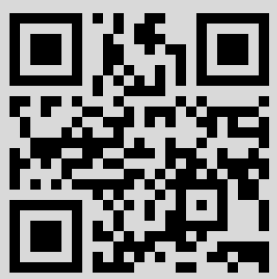




\title{
Гипотеза Римана \\ в терминах собственных чисел \\ специальных ганкелевых матриц
}

\author{
Ю. В. Матиясевич \\ Санкт-Петербургское отделение \\ Математического института им. В.А. Стеклова Российской академии наук \\ yumat@pdmi.ras.ru
}

\section{1. Введение}

Гипотеза Римана, подобно большинству великих проблем, имеет огромное количество эквивалентных переформулировок. В настоящей работе рассматривается одна из них, формулируемая в терминах собственных чисел ганкелевых матриц специального вида; элементы этих матриц задаются через коэффициенты разложения дзета-функции в ряд Тейлора в точке $s=0$. Гипотеза Римана предсказывает поведение абсолютных величин этих собственных чисел в среднем, но прямо не говорит ничего ни об отдельных собственных числах, ни об их распределении. Вычисления, выполненные для соответствующих матриц достаточно большого размера, прозволяют сформулировать предположения об их собственных числах, которые не следуют непосредственно из гипотезы Римана, но влекут ее.

Начало исследований упомянутых ганкелевых матриц было положено автором в [1]; двойственные к ним тёплицевы матрицы были введены в рассмотрение в [2]. Первые наблюдения над собственными числами этих матриц были представлены на Общеинститутском семинаре МИАН [3] и других семинарах и конференциях (см. [4]), они частично отражены в трудах конференций [5], [6], а также в преприте [7]. За продолжающимся исследованием этих матриц можно следить на [4].

\section{2. Подгипотезы гипотезы Римана}

Традиционно гипотеза Римана формулируется как утверждение о нулях дзета-функции Римана $\zeta(z)$, которая может быть определена при $\operatorname{Re}(z)>1$ рядом Дирихле:

$$
\zeta(z)=\sum_{n=1}^{\infty} \frac{1}{n^{z}} .
$$

Эта функция может быть аналитически продолжена на всю комплексную $z$-плоскость за исключением точки $z=1$, которая является единственным полюсом $\zeta(z)$.

Еще Л. Эйлер указал, что $0=\zeta(-2)=\zeta(-4)=\cdots$, и отрицательные четные числа ныне называют тривиальными нулями дзета-функции. Риман [8] установил, что других вещественных нулей нет, и высказал знаменитую гипотезу о расположении остальных, нетривиальных, нулей дзета-функции.

ГиПотезА Римана (версия 1). Все нетривиалъные нули функции $(z)$ лежат на критической прямой $\operatorname{Re}(z)=1 / 2$.

Работа была поддержана программой фундаментальных исследований Отделения математических наук РАН "Современные проблемы теоретической математики". 
Мы начнем с нескольких элементарных переформулировок гипотезы Римана.

Существует традиция (начало которой положено самим Риманом в его основополагающей работе [8]) избавляться от тривиальных нулей дзета-функции, рассматривая вместо нее целую функцию

$$
\xi(z)=\pi^{-z / 2}(z-1) \Gamma\left(1+\frac{z}{2}\right) \zeta(z)
$$

(мы используем современное обозначение для этой функции, сам Риман обозначал посредством $\xi(t)$ функцию, которая ныне традиционно обозначается как $\Xi(t))$. В $(2.2)$ полюса сомножителя $\Gamma(1+z / 2)$ гасят тривиальные нули $\zeta(z)$, а сомножитель $z-1$ гасит полюс этой

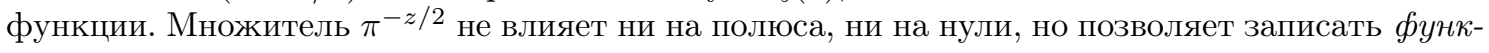
ииональное уравнение в виде

$$
\xi(z)=\xi(1-z)
$$

В этой работе мы не будем лишать дзета-функцию ее тривиальных нулей, а попытаемся использовать знание их местонахождения. Вместо $\xi(z)$ мы будем работать с целой функцией

$$
\zeta^{*}(z)=2(z-1) \zeta(z) \text {. }
$$

Мы могли бы не использовать множитель $z-1$ и/или использовать множитель $\pi^{-z / 2}$, что привело бы к несколько иным формулам, которым, возможно, будет посвящена отдельная статья. Множитель 2 в (2.4) приводит к равенству $\zeta^{*}(0)=1$, которое слегка упрощает некоторые последующие формулы.

Благодаря функциональному уравнению (2.3) мы можем ограничиться рассмотрением нулей, лежащих в одной полуплоскости.

ГиПотезА Римана (версия 2). Тривиальные нули $\mathrm{z}_{1}=-2, \mathrm{z}_{2}=-4, \ldots, \mathrm{z}_{n}=-2 n, \ldots$ являются единственными нулями функции $\zeta^{*}(z)$, лежащими в полуплоскости $\operatorname{Re}(z)<1 / 2$.

Полуплоскость является естественным объектом рассмотрения при работе с рядами Дирихле. Мы, однако, будем иметь дело с рядами Тейлора, для которых более естественными являются круги. Для перехода к ним мы сделаем замену переменных

$$
z=\frac{w}{w+1}, \quad w=\frac{z}{1-z} .
$$

При этом критическая прямая $\operatorname{Re}(z)=1 / 2$ становится критической окружностью $|w|=1$, полуплоскость $\operatorname{Re}(z)<1 / 2$ - открытым кругом $|w|<1$, а точки

$$
\mathrm{w}_{1}=\frac{\mathrm{z}_{1}}{1-\mathrm{z}_{1}}=-\frac{2}{3}, \quad \ldots, \quad \mathrm{w}_{n}=\frac{\mathrm{z}_{n}}{1-\mathrm{z}_{n}}=-\frac{2 n}{2 n+1}, \quad \ldots
$$

будут тривиальными нулями функции

$$
\widetilde{\zeta}(w)=\zeta^{*}\left(\frac{w}{w+1}\right) .
$$

ГиПотезА Римана (версия 3). Тривиальные нули (2.6) являются единственными нулями функиии $\widetilde{\zeta}(w)$, лежащими в открытом круге $\{w:|w|<1\}$.

$\mathrm{C}$ критической окружностью, полной нулей функции $\widetilde{\zeta}(w)$, работать непросто, и мы разобьем гипотезу Римана на бесконечную серию более слабых подгипотез.

ПоДГИПОТЕЗА $\mathrm{RH}_{n}$. Тривиальные нули $\mathrm{w}_{1}, \ldots, \mathrm{w}_{n}$ являются единственными нулями бункии $\widetilde{\zeta}(w)$, лежащими в замкнутом круге $\{w:|w| \leqslant(2 n+1) /(2 n+2)\}$.

Хотя каждая из этих подгипотез слабее гипотезы Римана, взятые вместе они, очевидно, ей эквивалентны.

ГиПотезА Римана (версия 4). Для каждого $n$ подгипотеза $\mathrm{RH}_{n}$ верна. 


\section{3. Приближения Паде и теорема Монтессу де Болора}

Для того чтобы “увидеть", где расположены наименьшие (по абсолютной величине) нули функции $\widetilde{\zeta}(w)$, мы можем приблизить ее рациональной функцией: пусть $P_{n, m}(w)$ и $Q_{n, m}(w)$ - многочлены такие, что

$$
\begin{aligned}
\widetilde{\zeta}(w) \approx \frac{P_{n, m}(w)}{Q_{n, m}(w)} & =\frac{1+p_{n, m, 1} w+\cdots+p_{n, m, n} w^{n}}{1+q_{n, m, 1} w+\cdots+q_{n, m, m} w^{m}} \\
& =\widetilde{\zeta}(w)+O\left(w^{k}\right),
\end{aligned}
$$

где $k$ имеет максимально возможное значение; поскольку в (3.1) в нашем распоряжении имеются $n+m$ коэффициентов

$$
p_{n, m, 1}, \ldots, p_{n, m, n}, \quad q_{n, m, 1}, \ldots, q_{n, m, m},
$$

то $k$ должно быть равно $n+m+1$ (отношение $P_{n, m}(w) / Q_{n, m}(w)$ называется апрроксимацией Паде типа $(n, m))$.

Будем говорить, что число $n$ является хорошим, если имеется замкнутый круг

$$
\{w:|w| \leqslant R\}
$$

содержащий (с учетом кратностей) ровно $n$ нулей функции $\widetilde{\zeta}(w)$. Теорема Монтессу де Болоpa [9] (см. также [10], [11]) говорит, что для любого хорошего $n$ нули многочлена $P_{n, m}(w)$ с ростом $m$ стремятся к нулям функции $\widetilde{\zeta}(w)$, лежащим в круге (3.4). Из подгипотезы $\mathrm{RH}_{n}$ следует, что $n$ является хорошим и, соответственно, при $m \rightarrow \infty$

$$
P_{n, m}(w) \rightarrow \prod_{k=1}^{n}\left(1-\frac{w}{\mathrm{w}_{n}}\right) .
$$

Мы будем иметь дело только с абсолютной величиной старшего коэффициента многочлена $P_{n, m}(w)$, в терминах которой можно сформулировать еще более слабые подгипотезы.

ПоДГИПОТЕзА $\mathrm{RH}_{n}^{w}$. Для $m \rightarrow \infty$

$$
\left|p_{n, m, n}\right| \rightarrow \mathrm{W}_{n}
$$

где

$$
\mathrm{W}_{n}=\prod_{k=1}^{n} \frac{1}{\left|\mathrm{w}_{n}\right|}=\prod_{k=1}^{n} \frac{2 k+1}{2 k}
$$

Даже эти, еще более слабые, подгипотезы, взятые вместе, влекут гипотезу Римана. Чтобы увидеть это, предположим, что она не верна, и пусть ऍ̌ - это наименьший по абсолютной величине нетривиальный нуль функции $\widetilde{\zeta}(w)$, нарушающий гипотезу Римана в версии 3. Круг (3.4) при $R=|\breve{\mathrm{w}}|$ содержит лишь конечное количество нулей функции $\widetilde{\zeta}(w)$, соответствующих нулям функции $\zeta^{*}(z)$ в прообразе этого круга на $z$-плоскости. Пусть это будут нули $\breve{\mathrm{w}}_{1}, \ldots, \widetilde{\mathrm{w}}_{n}$, занумерованные так, что $\left|\breve{\mathrm{w}}_{1}\right| \leqslant \cdots \leqslant\left|\breve{\mathrm{w}}_{n}\right|$. Согласно нашему выбору число $n$ является хорошим, и поэтому при $m \rightarrow \infty$

$$
P_{n, m}(w) \rightarrow \prod_{k=1}^{n}\left(1-\frac{w}{\check{\mathrm{w}}_{n}}\right)
$$


и, соответственно,

$$
\left|p_{n, m, n}\right| \rightarrow \prod_{k=1}^{n} \frac{1}{\left|\breve{\mathrm{w}}_{n}\right|} .
$$

Легко видеть, что $\left|\breve{\mathrm{w}}_{1}\right| \leqslant\left|\mathrm{w}_{1}\right|, \ldots,\left|\breve{\mathrm{w}}_{n-1}\right| \leqslant\left|\mathrm{w}_{n-1}\right|$ и $\left|\breve{\mathrm{w}}_{n}\right|<\left|\mathrm{w}_{n}\right|$. Следовательно,

$$
\prod_{k=1}^{n} \frac{1}{\left|\breve{\mathrm{w}}_{n}\right|}>\prod_{k=1}^{n} \frac{1}{\left|\mathrm{w}_{n}\right|}=\mathrm{W}_{n}
$$

что дает требуемое противоречие с (3.6).

ГиПотеЗА Римана (версия 5). Для каждого $n$ подгипотеза $\mathrm{RH}_{n}^{\mathrm{w}}$ верна.

Предыдущее рассуждение показывает, что в этой версии мы можем ограничиться хорошими значениями $n$.

\section{4. Определители и собственные числа}

Нетрудно понять, что коэффициенты (3.3) выражаются через коэффициенты разложения

$$
\widetilde{\zeta}(w)=1+\theta_{1} w+\cdots+\theta_{k} w^{k}+\cdots .
$$

Для упрощения последующих формул положим $\theta_{0}=1$ и $\theta_{k}=0$ при $k<0$. Якоби ([12], см. также [10]) дал явные выражения для $p_{n, m, n}$ через определители ганкелевых матриц

$$
M_{n, m}=(-1)^{n+m}\left(\begin{array}{cccc}
\theta_{n+m-1} & \theta_{n+m-2} & \ldots & \theta_{n} \\
\theta_{n+m-2} & \theta_{n+m-3} & \ldots & \theta_{n-1} \\
\vdots & \vdots & \ddots & \vdots \\
\theta_{n} & \theta_{n-1} & \ldots & \theta_{n-m+1}
\end{array}\right)
$$

а именно

$$
p_{n, m, n}=(-1)^{n+m+1} \frac{\operatorname{det}\left(M_{n, m+1}\right)}{\operatorname{det}\left(M_{n, m}\right)} .
$$

Если для некоторой константы $C$ при $m \rightarrow \infty$

$$
\left|p_{n, m, n}\right| \rightarrow C
$$

то из (4.3) следует, что

$$
\left|\operatorname{det}\left(M_{n, m}\right)\right|^{1 / m} \rightarrow C
$$

ПоДГИПОТЕЗА $\mathrm{RH}_{n}^{\mathrm{ww}}$. При $m \rightarrow \infty$

$$
\left|\operatorname{det}\left(M_{n, m}\right)\right|^{1 / m} \rightarrow \mathrm{W}_{n}
$$

Аналогично предыдущему, подгипотезы $\mathrm{RH}_{n}^{\mathrm{ww}}$, взятые вместе, влекут гипотезу Римана. Действительно, если бы она была не верна, то из (3.9), (3.10), (4.4) и (4.5) следовало бы, что для некоторого хорошего $n$ значение $\left|p_{n, m, n}\right|$ и, соответственно, значение $\left|\operatorname{det}\left(M_{n, m}\right)\right|^{1 / m}$ стремились бы к некоторой величине, превосходящей $\mathrm{W}_{n}$.

ГиПотезА Римана (версия 6). Для каждого $n$ подгипотеза $\mathrm{RH}_{n}^{\mathrm{ww}}$ верна.

Подгипотезу (4.6) можно пытаться доказывать индукцией по $n$. Мы знаем, что $-2 / 3$ является единственным нулем функции $\widetilde{\zeta}(z)$, лежащим в круге $|z|<3 / 4$, и, следовательно, 
подгипотеза $\mathrm{RH}_{1}^{\mathrm{ww}}$ верна. Для обоснования индукционного перехода было бы достаточно установить справедливость следующих подгипотез.

ПоДГИПОТЕЗА $\mathrm{RH}_{n}^{\mathrm{r}}$. При $m \rightarrow \infty$

$$
\frac{\left|\operatorname{det}\left(M_{n+1, m}\right)^{1 / m}\right|}{\left|\operatorname{det}\left(M_{n, m+1}\right)\right|^{1 /(m+1)}} \rightarrow \frac{\mathrm{W}_{n+1}}{\mathrm{~W}_{n}}=\frac{1}{\left|\mathrm{w}_{n+1}\right|}=\frac{2 n+3}{2 n+2} .
$$

Это дает еще одну переформулировку гипотезы Римана.

ГиПотезА Римана (версия 7). Для каждого $n$ подгипотеза $\mathrm{RH}_{n}^{\mathrm{r}}$ верна.

Обозначим через $\mu_{n, m, 1}, \mu_{n, m, 2}, \ldots, \mu_{n, m, m}$ собственные числа матрицы $M_{n, m}$ (они вещественны, поскольку матрица ганкелева). Две последние подгипотезы и две последние версии гипотезы Римана очевидным образом переформулируются в терминах этих собственных чисел.

ПоДГИПОТЕЗА $\mathrm{RH}_{n}^{\mathrm{ww}}$. $П р u m \rightarrow \infty$

$$
\frac{1}{m} \sum_{k=1}^{m} \ln \left|\mu_{n, m, k}\right| \rightarrow \ln \left(\mathrm{W}_{n}\right) .
$$

ГиПотезА Римана (версия 8). Для каждого $n$ подгипотеза $\mathrm{RH}_{n}^{\mathrm{ww}}{ }^{*}$ верна.

ПоДГИПотезА $\mathrm{RH}_{n}^{\mathrm{r}^{*}}$. При $m \rightarrow \infty$

$$
\frac{1}{m} \sum_{k=1}^{m} \ln \left|\mu_{n+1, m, k}\right|-\frac{1}{m+1} \sum_{k=1}^{m+1} \ln \left|\mu_{n, m+1, k}\right| \rightarrow-\ln \left(\left|\mathrm{w}_{n+1}\right|\right) .
$$

ГиПотеЗА Римана (версия 9). Для каждого $n$ подгипотеза $\mathrm{RH}_{n}^{\mathrm{r}^{*}}$ верна.

Для получения в дальнейшем еще одной переформулировки гипотезы Римана мы слегка преобразуем левую часть (4.9), а именно изменим сомножитель перед первой суммой - это возможно, поскольку левая часть в (4.8) имеет конечный предел.

ПоДГиПотезА $\mathrm{RH}_{n}^{\mathrm{r}^{* *}}$. При $m \rightarrow \infty$

$$
\frac{1}{m+1}\left(\sum_{k=1}^{m} \ln \left|\mu_{n+1, m, k}\right|-\sum_{k=1}^{m+1} \ln \left|\mu_{n, m+1, k}\right|\right) \rightarrow-\ln \left|\mathrm{w}_{n+1}\right| .
$$

ГиПотезА Римана (версия 10). Для каждого $n$ подгипотеза $\mathrm{RH}_{n}^{\mathrm{r} * *}$ верна.

\section{5. Визуальное представление собственных чисел}

Гипотеза Римана в версии 8 - это утверждение о поведении собственных чисел матриц (4.2) в среднем, однако она ничего не говорит, по крайней мере явно, о величине отдельных собственных чисел. Автор счел интересным провести вычисления, чтобы посмотреть, не будут ли наблюдаться какие-либо интересные закономерности в распределении значений этих собственных чисел. И действительно, оказалось, что при надлежащем графическом представлении рассматриваемые собственные числа демонстрируют интересные зрительные образы, которые позволяют сформулировать ряд гипотез. 
Каждое (мульти)множество $\left\{\mu_{n, m, 1}, \mu_{n, m, 2}, \ldots, \mu_{n, m, m}\right\}$ собственных чисел матрицы (4.2) мы будем называть $\mu$-спектром функции $\widetilde{\zeta}$ и обозначать через $\operatorname{Spec}_{n, m}^{\mu}(\widetilde{\zeta})$ или просто $\operatorname{Spec}_{n, m}^{\mu}$, если нам не будет требоваться одновременное рассмотрение аналогичных $\mu$-спектров других функций. Будем считать, что собственные числа перенумерованы так, что $\left|\mu_{n, m, k}\right| \leqslant$ $\left|\mu_{n, m, k+1}\right|$.

Спектры $\operatorname{Spec}_{n, m}^{\mu}$ содержат и очень большие, и очень маленькие (по абсолютной величине) числа, так что удобнее использовать логарифмическое масштабирование. Определим логарифмический $\mu$-спектр как (мульти)множество

$$
\operatorname{Spec}_{n, m}^{\ln \mu}=\left\{\ln (|\alpha|): \alpha \in \operatorname{Spec}_{n, m}^{\mu}\right\},
$$

элементы которого будем называть логарифмическими собственными числами.

Знаки собственных чисел несут полезную информацию, пропадающую в (5.1); чтобы сохранить ее, мы разобьем $\operatorname{Spec}_{n, m}^{\ln \mu}$ на положительный логарифмический $\mu$-спектр и отрицательный логарифмический $\mu$-спектр, определяемые следующим образом:

$$
\begin{aligned}
& \operatorname{Spec}_{n, m}^{\ln ^{+} \mu}=\left\{\ln (\alpha): \alpha \in \operatorname{Spec}_{n, m}^{\mu} \& \alpha>0\right\}, \\
& \operatorname{Spec}_{n, m}^{\ln ^{-} \mu}=\left\{\ln (-\alpha): \alpha \in \operatorname{Spec}_{n, m}^{\mu} \& \alpha<0\right\} .
\end{aligned}
$$

Изображая несколько логарифмических $\mu$-спектров на одном рисунке, мы будем сдвигать их друг относительно друга по вертикали, а именно собственное число $\alpha$ из $\operatorname{Spec}_{n, m}^{\mu}$ даст точку с координатами $(x, y)=(\ln |\alpha|, m)$.

Рисунки 1-4 показывают спектры $\operatorname{Spec}_{n, m}^{\ln \mu}$ для $n=1,2,3,4$ (рис. для $n=5,6$ приведены в [13]). Прежде всего можно заметить, что спектры $\operatorname{Spec}_{n, m}^{\ln ^{+} \mu}$ и $\operatorname{Spec}_{n, m+1}^{\ln ^{+} \mu}$ близки друг другу, и аналогичная близость имеет место для спектров $\operatorname{Spec}_{n, m}^{\ln ^{-} \mu}$ и $\mathrm{Spec}_{n, m+1}^{\ln ^{-} \mu}$. Вследствие этого мы наблюдаем некоторые "траектории" и, считая $m$ (дискретным) временем, можем вообразить "частицы", движущиеся по ним.

Чтобы ввести это понятие формально, мы перенумеруем логарифмические собственные числа двумя способами, а именно слева направо и наоборот. Обозначим через $N_{n, m}^{+}$и $N_{n, m}^{-}$ количества (с учетом возможной кратности) элементов в (5.2) и (5.3) соответственно, и пусть

$$
\operatorname{Spec}_{n, m}^{\ln ^{+} \mu}=\left\{\mu_{n, m, 1}^{+<}, \ldots, \mu_{n, m, N_{n, m}^{+}}^{+<}\right\}=\left\{\mu_{n, m, 1}^{+>}, \ldots, \mu_{n, m, N_{n, m}^{+}}^{+>}\right\}
$$

причем $\mu_{n, m, k}^{+<} \leqslant \mu_{n, m, k+1}^{+<}, \mu_{n, m, k}^{+>} \geqslant \mu_{n, m, k+1}^{+>}$, и, аналогично,

$$
\operatorname{Spec}_{n, m}^{\ln ^{-} \mu}=\left\{\mu_{n, m, 1}^{-<}, \ldots, \mu_{n, m, N_{n, m}^{-}}^{-<}\right\}=\left\{\mu_{n, m, 1}^{->}, \ldots, \mu_{n, m, N_{n, m}^{-}}^{->}\right\}
$$

причем $\leqslant \mu_{n, m, k}^{-<} \leqslant \mu_{n, m, k+1}^{-<}, \geqslant \mu_{n, m, k}^{->} \geqslant \mu_{n, m, k+1}^{->}$.

По верхнему индексу мы можем различать четыре типа частиц: $\pi_{n, k}^{+<}, \pi_{n, k}^{-<}, \pi_{n, k}^{+>}$и $\pi_{n, k}^{->}$. В момент времени $m$ эти четыре частицы имеют координаты $\mu_{n, m, k}^{+<}, \mu_{n, m, k}^{+>}, \mu_{n, m, k}^{-<}$и $\mu_{n, m, k}^{->}$ соответственно. Частицы $\pi_{n, k_{1}}^{+<}$и $\pi_{n, k_{2}}^{+>}$имеют равные координаты при условии, что $k_{1}+k_{2}=$ $N_{n, m}^{+}+1$; аналогично равные координаты имеют частицы $\pi_{n, k_{1}}^{-<}$и $\pi_{n, k_{2}}^{->}$при условии, что $k_{1}+k_{2}=N_{n, m}^{-}+1$. Из каждой пары частиц с равными координатами одна в итоге стремится $\mathrm{k}-\infty$, а другая $-\mathrm{k}+\infty$.

Частицы $\pi_{n, k}^{+<}$и $\pi_{n, k}^{-<}$, которые с ростом $m$ стремятся к $-\infty$, были названы в [1] электронами. Это название обязано следующему наблюдению, которое можно сделать по рис. 1-4, а еще нагляднее - по анимации [4]. А именно, траектории электронов, соответствующие положительным и отрицательным собственным числам, перемежаются друг с другом, осциллируют, но не пересекаются (отталкиваются друг от друга), т.е. ведут себя подобно одноименно заряженным частицам. 


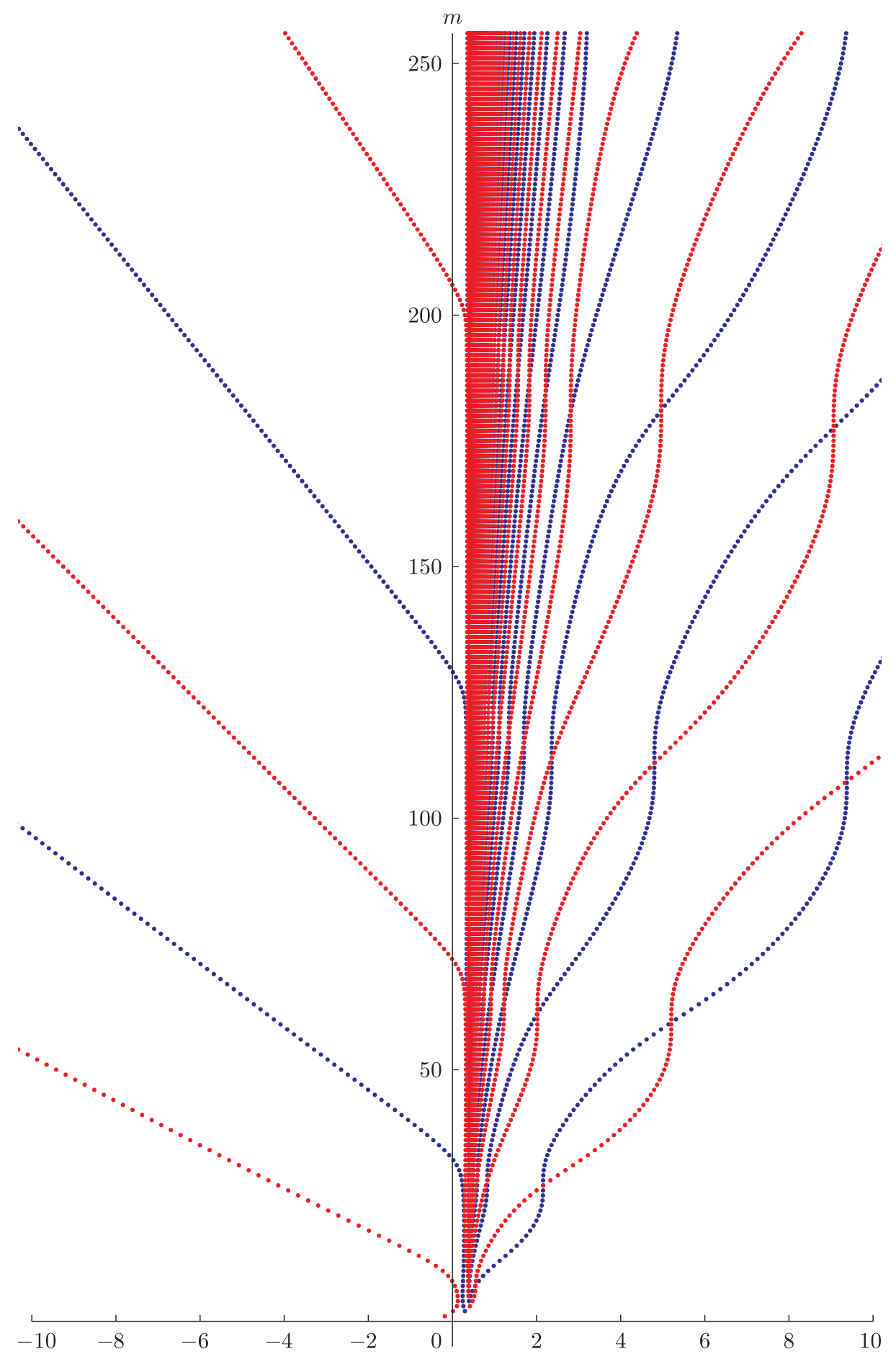

Рис. 1. Положительные (красные круги) и отрицательные (синие круги) логарифмические $\mu$-спектры $\operatorname{Spec}_{1, m}^{\ln ^{ \pm} \mu}, m=1, \ldots, 256$ 


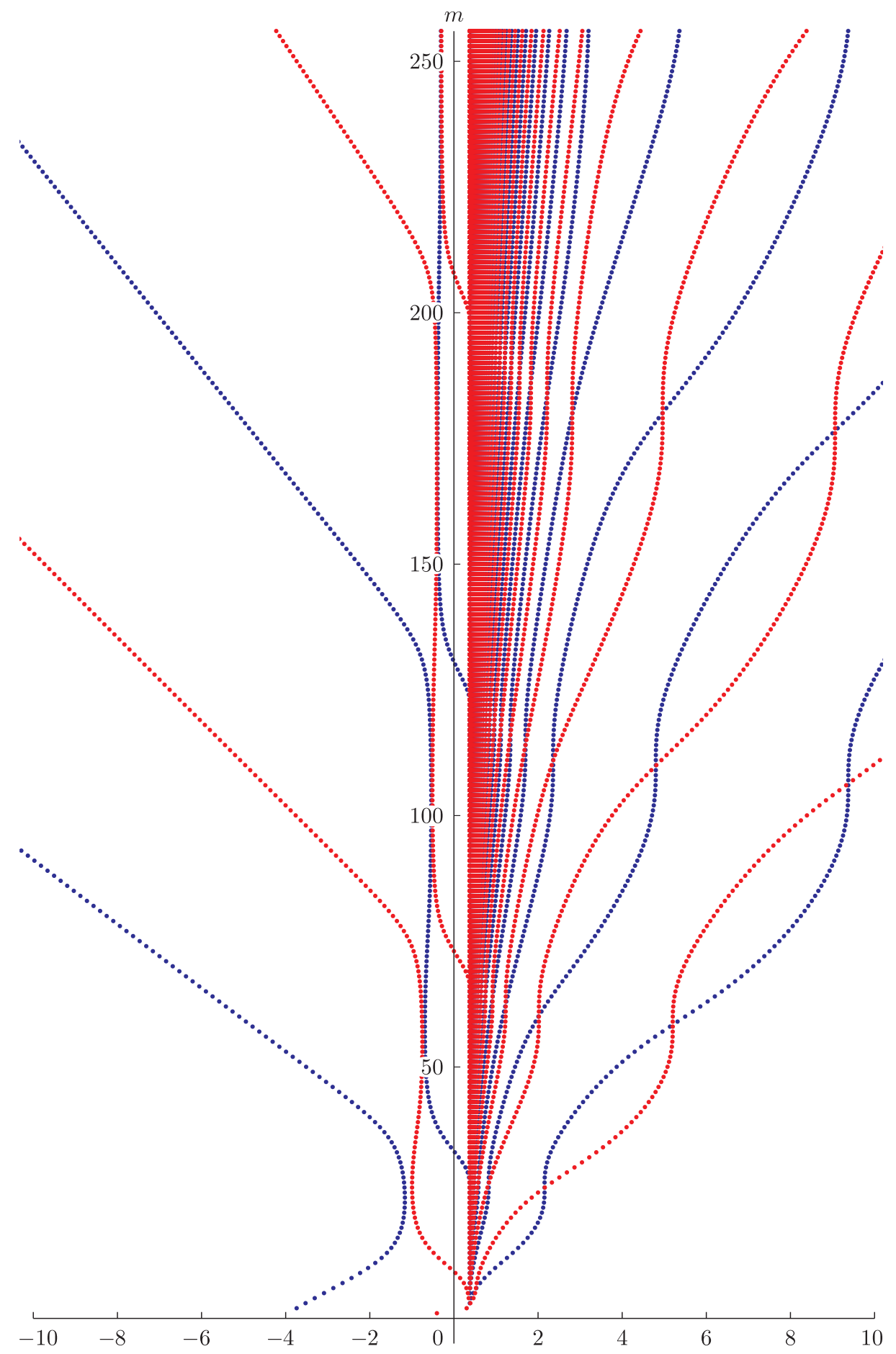

Рис. 2. Положительные (красные круги) и отрицательные (синие круги) логарифмические $\mu$-спектры $\operatorname{Spec}_{2, m}^{\ln ^{ \pm} \mu}, m=1, \ldots, 256$ 


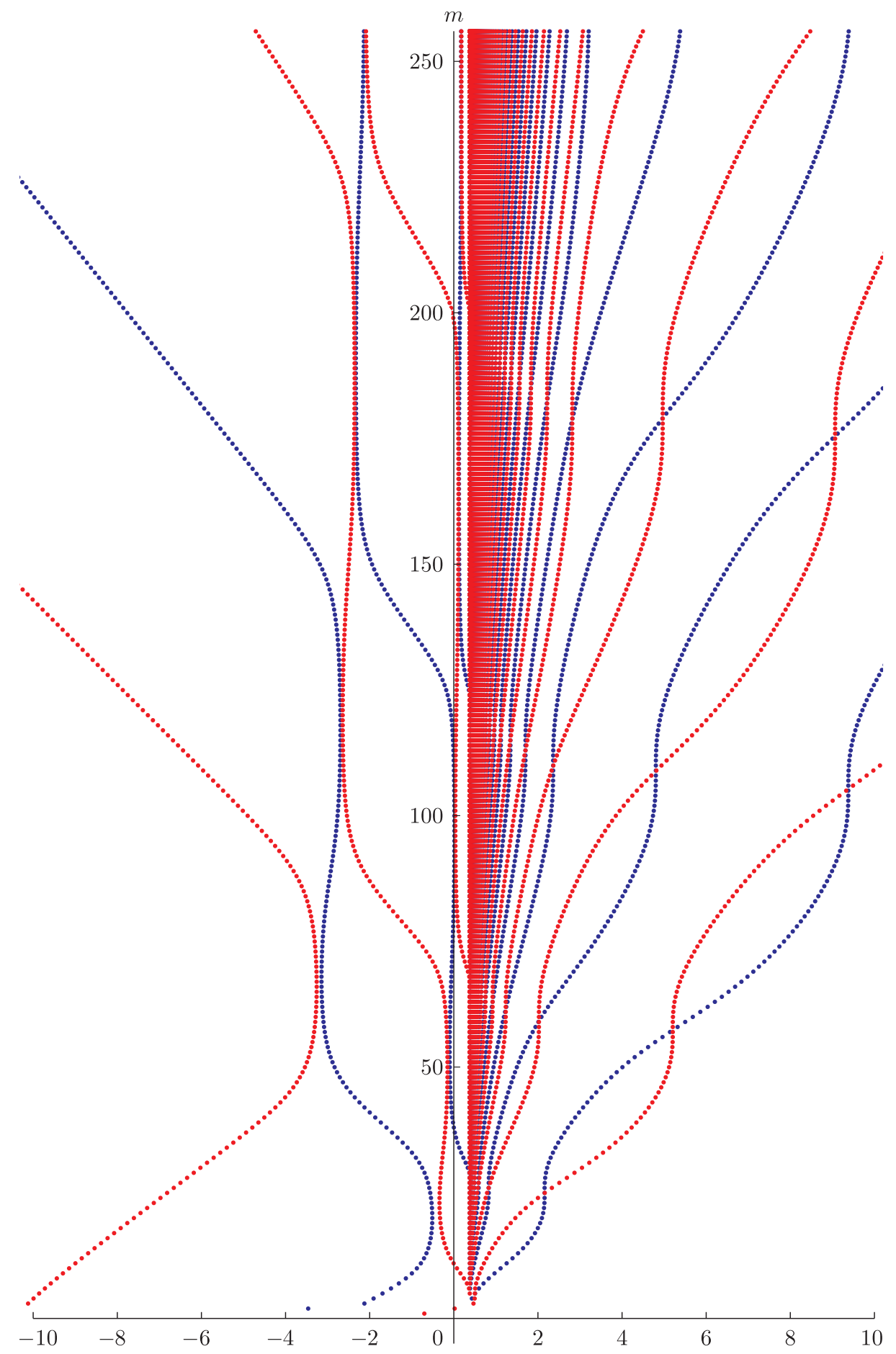

Рис. 3. Положительные (красные круги) и отрицательные (синие круги) логарифмические $\mu$-спектры $\operatorname{Spec}_{3, m}^{\ln ^{ \pm} \mu}, m=1, \ldots, 256$ 


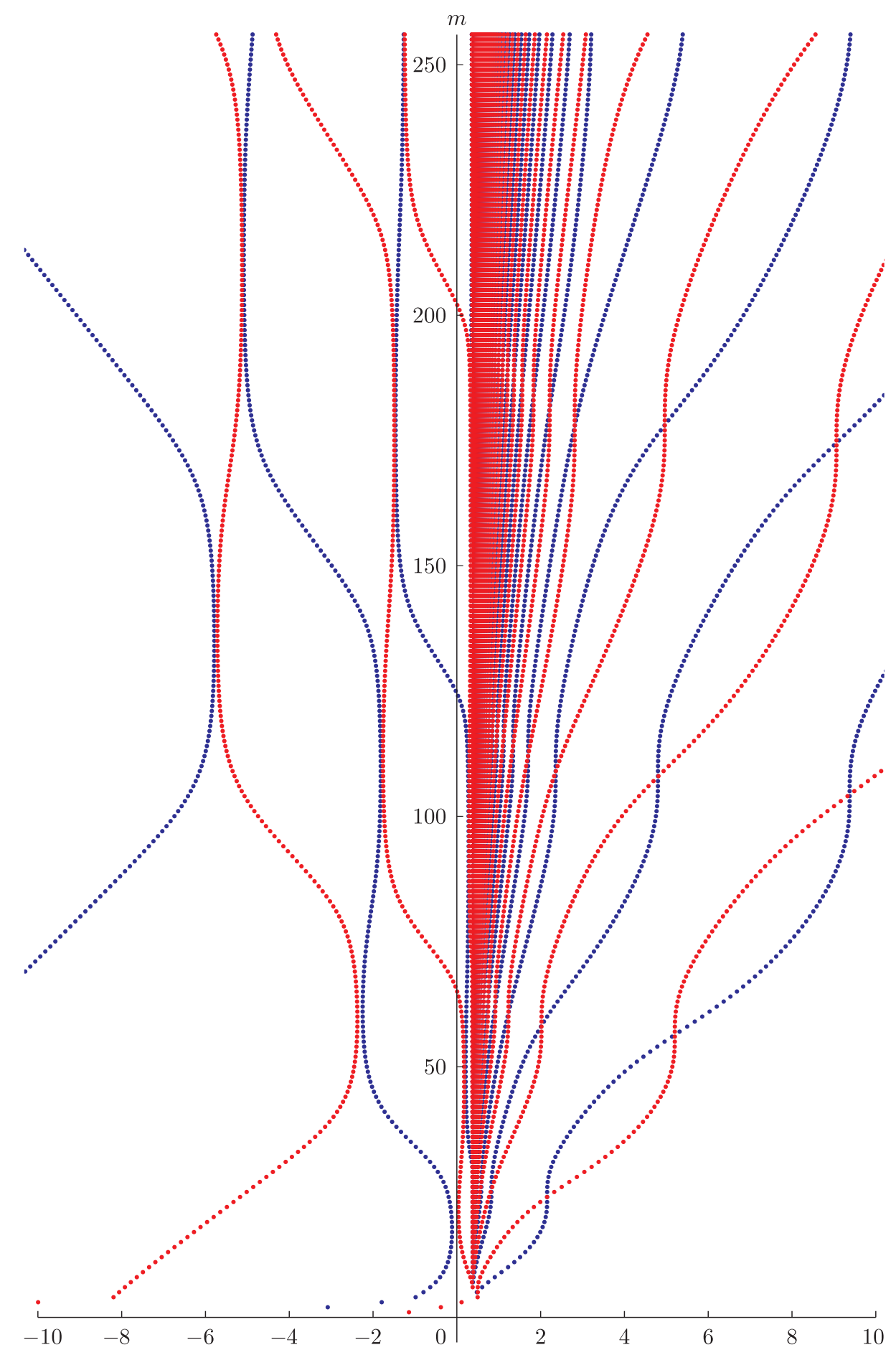

Рис. 4. Положительные (красные круги) и отрицательные (синие круги) логарифмические $\mu$-спектры $\operatorname{Spec}_{4, m}^{\ln ^{ \pm}} \mu, m=1, \ldots, 256$ 
В отличие от электронов, траектории частиц $\pi_{n, k}^{+>}$и $\pi_{n, k}^{->}$, которые с ростом $m$ стремятся к $+\infty$, непрерывно пересекаются, образуя на рис. 1-4 характерные косички. В [1] эти частицы были названы поездами

\section{6. Дальнейшие переформулировки и новые предположения}

Рисунки 1-4 показывают, что, изменив направление движения несколько раз, каждый электрон начинает двигаться со стабилизирующейся скоростью (графически это означает, что траектории приближаются к прямым линиям). Численные данные [13] позволяют предположить, что эта скорость замечательным образом связана с тривиальными нулями и поведением дзета-функции в них.

ПРЕДПОЛОЖЕНИЕ А. Для всех $n$ и $k$ существует рациональное число $A_{n, k}$ такое, что nрu $m \rightarrow \infty$

$$
\mu_{n, m, k}=(-1)^{n+k}\left(Y_{n, k}+o(1)\right)\left(-\mathrm{w}_{n+k}\right)^{m}
$$

где

$$
Y_{n, k}=\left.A_{n, k} \frac{d}{d w} \widetilde{\zeta}(w)\right|_{w=\mathrm{w}_{n+k}} .
$$

Более конкретно, для $n=0$ следует ожидать, что

$$
A_{0, k}=\frac{(4 k-1) ! !(4 k+1) ! !}{2^{2 k-2}(2 k+1)^{2}((k-1) !)^{2}((2 k+1) ! !)^{2}} .
$$

При $n>0$ величину $A_{n, k}$ удалось предсказать лишь для отдельных значений $n$ и $k$ :

$$
\begin{aligned}
& A_{1,1}=\frac{63}{23}=\frac{3^{2} \times 7}{23}, \\
& A_{1,2}=\frac{325611}{10336}=\frac{3^{2} \times 11^{2} \times 13 \times 23}{2^{5} \times 17 \times 19}, \\
& A_{2,1}=\frac{405405}{29776}=\frac{3^{4} \times 5 \times 7 \times 11 \times 13}{2^{4} \times 1861}, \\
& A_{3,1}=\frac{23562}{325}=\frac{2 \times 3^{2} \times 7 \times 11 \times 17}{5^{2} \times 13}, \\
& A_{4,1}=\frac{220061716875}{539180032}=\frac{3^{2} \times 5^{4} \times 7 \times 11^{3} \times 13 \times 17 \times 19}{2^{10} \times 526543} .
\end{aligned}
$$

Указанные выше ожидаемые значения $A_{n, k}$ были получены следующим образом. Вычислялось с большой точностью значение $\mu_{n, m, k}$ для достаточно большого значения $m$, а затем отношение

$$
\frac{(-1)^{n+k} \mu_{n, m, k}}{\left.(-w)^{m} \frac{d}{d w} \widetilde{\zeta}(w)\right|_{w=\mathrm{w}_{n+k}}}
$$

разлагалось в цепную дробь. Например, при $n=1, m=511, k=1$ значение (6.4) равно

$$
2.739130434782610541268416612253 \ldots=[2,1,2,1,5,1024242810887,2,1,1, \ldots] \text {. }
$$

Из метрической теории цепных дробей известно, что больша́я величина неполного частного у "случайного" вещественного числа маловероятна, а это позволяет предположить, что $A_{n, k}$ равно конечной цепной дроби $[2,1,2,1,5]$. Косвенным подтверждением правильности подобного предположения является получение той же конечной цепной дроби при других больши́х 
значениях $m$, а также наличие у числителей и знаменателей получаемой конечной дроби большого количества небольших простых делителей. Другие численные данные, подсказавшие сделанный выбор значений $A_{n, k}$, приведены в [13].

Предположение А представляется независимым от гипотезы Римана - не видно, ни как вывести его из этой гипотезы, ни как получить ее в виде следствия этого предположения. Однако, считая предположение А выполненным, мы можем дать еще одну переформулировку гипотезы Римана и высказать предположение, которое (формально) ее сильнее.

А именно, согласно (6.2) при $k>1$ скорости электронов, соответствующих собственным числам $\mu_{n, m, k}$ и $\mu_{n+1, m, k-1}$, асимптотически различаются на константу. Это подсказывает следующее перегруппирование слагаемых в левой части (4.10):

$$
\begin{aligned}
& \frac{1}{m+1}\left(\sum_{k=1}^{m} \ln \left|\mu_{n+1, m, k}\right|-\sum_{k=1}^{m+1} \ln \left|\mu_{n, m+1, k}\right|\right) \\
& \quad=-\frac{\ln \left|\mu_{n, m+1,1}\right|}{m+1}+\frac{1}{m+1} \sum_{k=1}^{m}\left(\ln \left|\mu_{n+1, m, k}\right|-\ln \left|\mu_{n, m+1, k+1}\right|\right) .
\end{aligned}
$$

Согласно предположению А первое слагаемое в правой части (6.6) стремится при $m \rightarrow \infty$, к тому же значению, а именно к $-\ln \left|\mathrm{w}_{n+1}\right|$, к которому согласно (4.10) стремится и левая часть (6.6). Иными словами, предположение А вместе с гипотезой Римана влекут необходимость обнуления в пределе второго слагаемого в правой части (6.6).

ПреДПоложЕниЕ В (слабая форма). Для каждого $n$ nрu $m \rightarrow \infty$

$$
\sum_{k=1}^{m}\left(\ln \left|\mu_{n+1, m, k}\right|-\ln \left|\mu_{n, m+1, k+1}\right|\right)=o(m) .
$$

Аналогично, предположения А и В, вместе взятые, влекут гипотезу Римана.

Проведенные вычисления указывают на следующий характер поведения левой части в (6.7).

ПрЕДПОЛОЖЕНИЕ В (сильная форма). Для каждого $n$ существует рачионалъное число $B_{n}$ такое, что при $m \rightarrow \infty$

$$
\sum_{k=1}^{m}\left(\ln \left|\mu_{n+1, m, k}\right|-\ln \left|\mu_{n, m+1, k+1}\right|\right) \rightarrow \ln \left(B_{n}\right) .
$$

Для небольших $n$ удается указать ожидамые значения $B_{n}$ :

$$
\begin{aligned}
& B_{0}=\frac{5}{9}=\frac{5}{3^{2}}, \\
& B_{1}=\frac{7}{115}=\frac{7}{5 \times 23}, \\
& B_{2}=\frac{143}{65135}=\frac{11 \times 13}{5 \times 7 \times 1861}, \\
& B_{3}=\frac{187}{4606875}=\frac{11 \times 17}{3^{4} \times 5^{4} \times 7 \times 13}, \\
& B_{4}=\frac{4199}{9122357475}=\frac{17 \times 19}{3^{2} \times 5^{2} \times 7 \times 11 \times 526543}, \\
& B_{5}=\frac{185725}{52544656365201}=\frac{5^{2} \times 17 \times 19 \times 23}{3^{2} \times 7 \times 11 \times 13 \times 19793 \times 294673} .
\end{aligned}
$$




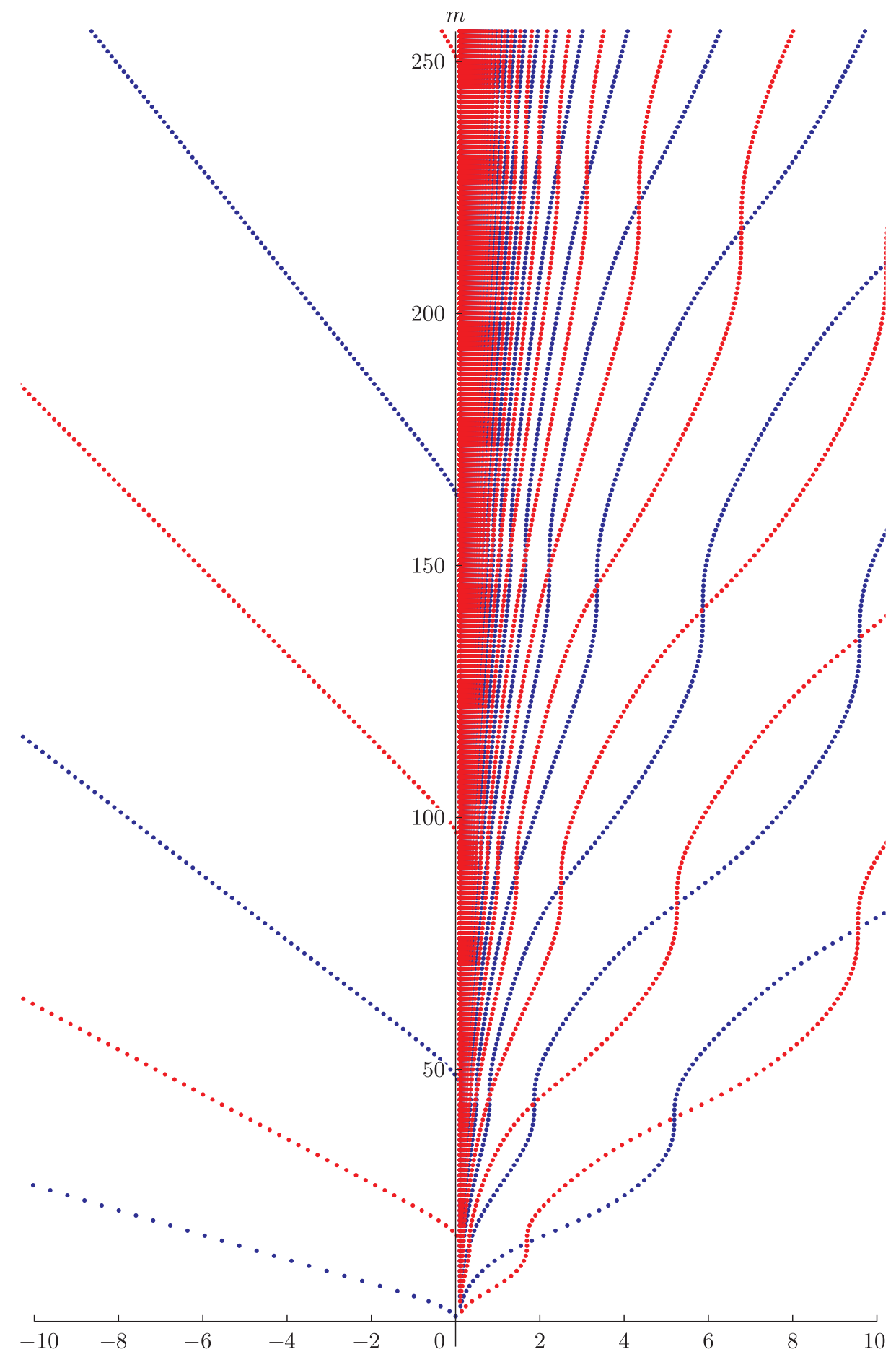

Рис. 5. Положительные (красные круги) и отрицательные (синие круги) логарифмические $\mu$-спектры $\operatorname{Spec}_{0, m}^{\ln ^{ \pm}} \mu\left(\zeta_{\text {Triv }}\right), m=1, \ldots, 256$ 
После обнаружения связи электронов с тривиальными нулями, выраженной в предположении А, может возникнуть желание связать поезда с па́рами комплексно сопряженных нетривиальных нулей. Такой связи, однако, скорее всего нет. Это показывает рассмотрение аналогов матриц (4.2), построенных для фунции $\zeta_{\operatorname{Triv}}(z)=1 / \Gamma(1+z / 2)$ вместо $\zeta(z)$. Единственными нулями этой функции являются тривиальные нули дзета-функции, тем не менее на рис. 5 отчетливо видны такие же косички. Про электроны функции $\zeta_{\operatorname{Triv}}(z)$ можно выдвинуть аналог предположения $\mathrm{A}$, причем с теми же константами $A_{n, k}$.

На рис. 1-4 видно, что поезда имеют точку сгущения. Для ее формального описания предположим, что каждый элемент спектра $\operatorname{Spec}_{n, m}^{\ln \mu}$ имеет вес $1 / m$, и рассмотрим соответствующую функцию распределения

$$
F_{n, m}(x)=\sum_{\ln \left|\mu_{n, m, k}\right| \leqslant x} \frac{1}{m}
$$

В этих обозначениях подгипотезу $\mathrm{RH}_{n}^{\mathrm{ww} *}$ можно переписать следующим образом.

ПоДГИПотеЗА $\mathrm{RH}_{n}^{\mathrm{ww}}{ }^{* *}$. При $m \rightarrow \infty$

$$
\int_{-\infty}^{+\infty} x d F_{n, m}(x) \rightarrow \ln \left(\mathrm{W}_{n}\right)
$$

Вычисления (см., например, [13]) позволяют ожидать существование предельного распределения, удивительным образом независящего от $n$.

ПреДПОЛОжЕНИЕ С. Существует непрерывная функиия $F(x)$ такая, что для каждого $n$ и каждого $x$ при $m \rightarrow \infty$

$$
F_{n, m}(x) \rightarrow F(x),
$$

причем $F(x)=0$ при $x<C$ и $F(x)>0$ при $x>C$, где

$$
C=\ln (\widetilde{\zeta}(1))=\ln \left(-\zeta\left(\frac{1}{2}\right)\right)=0.378679220 \ldots
$$

Предполагаемый вид $F(x)$ показан на рис. 6 .

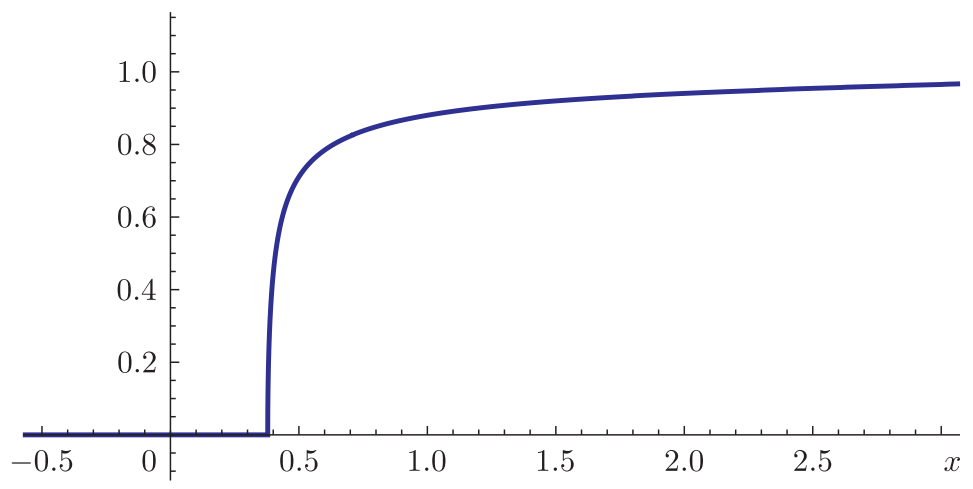

Рис. 6. Ожидаемый график $F(x)$ 


\section{Список литературы}

[1] Yu. Matiyasevich, Hidden Life of Riemann's Zeta Function 2. Electrons and Trains, 2007, arXiv: 0709.0028.

[2] Yu. Matiyasevich, Hidden Life of Riemann's Zeta Function 1. Arrow, Bow, and Targets, 2007, arXiv: 0707.1983.

[3] Ю. В. Матиясевич, Тайная жизнъ дзета-функции Римана, Выступление на Общеинститутском семинаре «Математика и ее приложения» Математического института им. В. А. Стеклова Российской академии наук, 2008, http://www.mathnet.ru/present/260.

[4] Yu. V. Matiyasevich, Hidden Life of Riemann's Zeta Function, http://logic.pdmi.ras.ru/ yumat/ personaljournal/zetahiddenlife.

[5] Yu. Matiyasevich, "Riemann's zeta function: Some computations and conjectures", Proceedings of Conference on Algorithmic Number Theory (Turku, 2007), TUCS General Publ. Ser., 46, Turku, 2007, 87-112, http://logic.pdmi.ras.ru/〜yumat/personaljournal/zetahiddenlife/talks/ turku2007/Matiyasevich_2007.pdf.

[6] Yu. Matiyasevich, "Riemann's zeta function: More computations and conjectures", Voronoï's Impact on Modern Science, Proceedings of the 4th International Conference on Analytical Number Theory and Spacial Tesselations, Book 4, Vol. 1 (Kyiv, 2008), Kyiv, 2008, 2-11, http://logic.pdmi.ras.ru/ yumat/personaljournal/zetahiddenlife/talks/kiev_2008/Matiyasevich_2008.pdf.

[7] Yu. V. Matiyasevich, The Riemann Hypothesis and Eigenvalues of Related Hankel Matrices. I, Preprint POMI 14-03, 2014, http://www.pdmi.ras.ru/preprint/2014/14-03.html.

[8] B. Riemann, "Über die Anzahl der Primzahlen unter einer gegebenen Grösse", Monatsber. Berliner Akad., 1859; Gesammelte Werke, Teubner, Leipzig, 1892; Dover Books, New York, 1953; http:// www.maths.tcd.ie/pub/HistMath/People/Riemann/Zeta/EZeta.pdf.

[9] R. de Montessus de Ballore, "Sur le fraction continues algébriques", Bull. Soc. Math. France, 30 (1902), $28-36$.

[10] G. A. Baker, Jr., Essentials of Padé Approximations, Academic Press, New York, 1975.

[11] E. B. Saff, "An extension of Montessus de Ballore's theorem on the convergence of interpolating rational functions", J. Approx. Theory, 6:1 (1972), 63-67.

[12] C. G. J. Jacobi, "Über die Darstellung einer Reihe Gegebner Werthe durch eine Gebrochne Rationale Function", J. Reine Angew. Math., 30 (1846), 127-156.

[13] Yu. V. Matiyasevich, Structure of Eigenvalues and Eigenvectors of Certain Almost Triangular Hankel Matrices, Выступление на 7-й Петербургской конференции по спектральной теории, 2015, http:// logic.pdmi.ras.ru/ yumat/talks/birman_2015. 